\title{
Lattice Problems in NP $\cap$ coNP
}

\author{
Dorit Aharonov* $\quad$ Oded Regev ${ }^{\dagger}$
}

September 8, 2005

\begin{abstract}
We show that the problems of approximating the shortest and closest vector in a lattice to within a factor of $\sqrt{n}$ lie in NP intersect coNP. The result (almost) subsumes the three mutually-incomparable previous results regarding these lattice problems: Banaszczyk [7], Goldreich and Goldwasser [14], and Aharonov and Regev [2]. Our technique is based on a simple fact regarding succinct approximation of functions using their Fourier series over the lattice. This technique might be useful elsewhere we demonstrate this by giving a simple and efficient algorithm for one other lattice problem (CVPP) improving on a previous result of Regev [26]. An interesting fact is that our result emerged from a "dequantization" of our previous quantum result in [2]. This route to proving purely classical results might be beneficial elsewhere.
\end{abstract}

\section{Introduction}

A lattice is the set of all integer combinations of $n$ linearly independent vectors $v_{1}, \ldots, v_{n}$ in $\mathbb{R}^{n}$. These vectors are known as a basis of the lattice. The study of lattices originated some 200 years ago by Gauss [12], who gave an algorithm to find the shortest vector in a two-dimensional lattice. Since then, lattices have been shown to be pervasive in mathematics, and many different problems can be phrased as questions about lattices, such as integer programming [18], factoring polynomials with rational coefficients [23], integer relation finding [16], integer factoring and Diophantine approximation [28]. Recently, the study of lattices gained a lot of attention in the computer science community due to the fact that lattice problems were shown by Ajtai [3] to possess a particularly desirable property for cryptography: worst-case to average-case reducibility.

Two lattice problems have been widely studied. The first is the Shortest Vector Problem (SVP): given a basis $v_{1}, \ldots, v_{n}$ of a lattice, find the shortest nonzero lattice point in the Euclidean norm. The second is the Closest Vector Problem (CVP): given a basis $v_{1}, \ldots, v_{n}$ of a lattice and a target vector $v \in \mathbb{R}^{n}$ find the closest lattice point to $v$ in the Euclidean norm. Both problems are known to be NP-complete $[4,30]$. In light of this, and the importance of lattice problems in mathematics, a very interesting question is the study of the approximation version of these problems. The parameter of interest here is the factor of approximation $\beta$. The problem GapSVP $\beta$ is the following: Given a basis $v_{1}, \ldots, v_{n}$, decide whether the $l_{2}$ norm of the shortest nonzero vector in the lattice is at most 1 or larger than $\beta$. The problem $\operatorname{GapCVP}_{\beta}$ is: Given a basis $v_{1}, \ldots, v_{n}$ and an extra vector $v \in \mathbb{R}^{n}$, decide whether the distance of $v$ from the lattice is at most 1 or larger than $\beta$. The best inapproximability result for CVP is due to Dinur et al. [10] where it is shown that GapCVP ${ }_{\beta}$ with $\beta=n^{c / \log \log n}$ is NP-hard for some $c>0$. For SVP, Khot [20] recently showed that for any $\varepsilon>0$ obtaining approximation factors below $2^{(\log n)^{1 / 2-\varepsilon}}$ is hard unless NP $\subseteq$ BPTIME $\left(2^{\text {poly }(\log n)}\right)$; this improves on a previous result of Micciancio [24]. The best probabilistic polynomial time approximation algorithm

\footnotetext{
${ }^{*}$ School of Computer Science and Engineering, The Hebrew University, Jerusalem, Israel. doria@cs.huji.ac.il. Research supported by an Alon Fellowship, and ISF grant 032-9738.

${ }^{\dagger}$ Department of Computer Science, Tel-Aviv University, Tel-Aviv 69978, Israel. Work supported by the Israel Science Foundation, an Alon Fellowship, and the Army Research Office grant DAAD19-03-1-0082.
} 
due to Ajtai et al. [6] obtains a $2^{O(n \log \log n / \log n)}$-approximation factor for both problems; it is based on the deterministic polynomial time $2^{O\left(n(\log \log n)^{2} / \log n\right)}$-approximation algorithm by Schnorr [27].

The complexity of lattice problems in the range of polynomial approximation factors is of particular interest. For example, Ajtai's seminal work [3] is based on the hardness of approximation in this region (see also $[5,25])$. A sequence of incomparable results gave upper bounds on the complexity of lattice problems in the polynomial approximation region. Banaszczyk [7] showed that GapCVP ${ }_{n}$ is in NP $\cap$ coNP, improving on the previous result of GapCVP $n^{1.5} \in N P \cap$ coNP by Lagarias, Lenstra and Schnorr [22]. We note that containment in NP is trivial, and the difficult part is showing the containment in coNP, i.e., showing the existence of a succinct proof that a vector is far from any lattice point. Goldreich and Goldwasser [14] gave an upper bound on the complexity of the harder problem GapCVP $\sqrt{n / \log n}$, but their upper bound is weaker: they showed containment in NP $\cap$ coAM, which means that instead of showing the existence of a succinct proof that a vector is far from any lattice point, they gave an interactive proof of two rounds to that effect. In another result, the current authors showed [2] that a certain special case of GapCVP $\sqrt{n}$ is in NP $\cap \operatorname{coQMA}$, where the latter class is the quantum analogue of coNP. Essentially, this says that there exists a succinct quantum proof that a vector is far from the lattice. See [2] for more details.

In this paper we prove the following theorem, which essentially subsumes all three results mentioned above.

Theorem 1.1 There exists $c>0$ such that $\mathrm{GapCVP}_{c \sqrt{n}}$ is in NP $\cap$ coNP.

Of the three results, the only result that Theorem 1.1 does not completely subsume is that of Goldreich and Goldwasser [14]. Indeed, for gaps between $\sqrt{n / \log n}$ and $\sqrt{n}$ our result does not apply, and so containment in NP $\cap$ coNP is not known to hold.

There is a known approximation preserving reduction from GapSVP to GapCVP [15], which we include for completeness in Appendix A. Using this reduction, we obtain the following corollary.

Corollary 1.2 There exists $c>0$ such that GapSVP ${ }_{c \sqrt{n}}$ is in NP $\cap$ coNP.

We summarize the current complexity of lattice problems as a function of the approximation ratio $\beta$ in Figure 1.

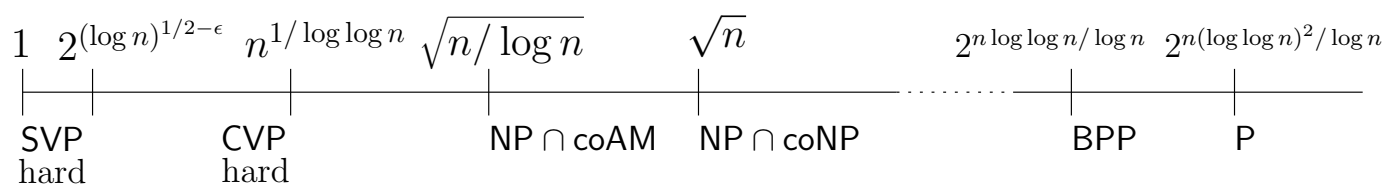

Figure 1: The complexity of lattice problems (some constants omitted)

\subsection{Proof Overview}

As mentioned before, the containment in NP is trivial and it suffices to prove, e.g., that GapCVP $100 \sqrt{n}$ is in coNP. To show this we construct an NP verifier that given a polynomial witness, verifies that $v$ is far from the lattice. There are three steps to this proof.

\section{Define $f$}

In this part we define a function $f: \mathbb{R}^{n} \rightarrow \mathbb{R}^{+}$that is periodic over the lattice $L$, i.e., for all $x \in \mathbb{R}^{n}$ and $y \in L$ we have $f(x)=f(x+y)$. For any lattice $L$, the function $f$ satisfies the following two properties: it is non-negligible (i.e., larger than some $1 / \operatorname{poly}(n)$ ) for any point that lies within distance $\sqrt{\log n}$ from a lattice point, and is exponentially small at distance $\geq \sqrt{n}$ from the lattice. Note that $f(v)$ indicates whether $v$ is far or close to the lattice. 


\section{Encode $f$}

We show that there exists a succinct description (which we denote by $W$ ) of a function $f_{W}$ that approximates $f$ at any point in $\mathbb{R}^{n}$ to within polynomially small additive error. We now use $W$ as the witness in the NP proof.

\section{Verify $f$}

We construct an efficient NP verifier that, given a witness $W$, verifies that $v$ is far from the lattice. The verifier verifies first that $f_{W}(v)$ is small, and also that $f_{W}(x) \geq 1 / 2$ for any $x$ that is close to the lattice.

Step 1 The function $f$ already appeared in [7], and in fact, the two properties mentioned in Step 1 were already proven there. The function is defined as a sum of Gaussians centered around each lattice point.

Step 2 This step is the core of the proof. Here we show that the function $f$ can be approximated pointwise by a polynomial size circuit with only an inverse polynomial additive error. A naive attempt would be to store $f$ 's values on some finite subset of its domain, and use these points for approximation on the rest of the domain. However, it seems that for this to be meaningful, we would have to store an exponential number of points.

Instead, we consider the Fourier series of $f$, denoted $\hat{f}$. By definition, the domain of $\hat{f}$ is the dual lattice (defined as the set of all points in $\mathbb{R}^{n}$ with integer inner product with all lattice points). It turns out that $\hat{f}$ has a useful property: it is a probability measure over the dual lattice. In other words, it is a non-negative function and the sum of all its values is 1 . This allows us to view $f$ as an expectation of a random variable, and so by the Chernoff-Hoeffding bound, polynomially many samples from the distribution on the dual lattice given by $\hat{f}$ would suffice. This leads us to the following lemma. We will later define $\ell$ as some polynomial in $n$ and $L_{\ell}$ as a very fine grid in $\mathbb{R}^{n}$. For now, one can think of the lemma as applying to any $x \in \mathbb{R}^{n}$ and not only to $x \in L_{\ell}$.

Lemma 1.3 (The Pointwise Approximation Lemma) Let $L$ be an $n$-dimensional lattice, and let $f$ be a function from $\mathbb{R}^{n}$ to $\mathbb{R}$ that is periodic over $L$ and whose Fourier series $\hat{f}$ is a probability measure over the dual lattice $L^{*}$. For any constant $c>0$ define $N$ to be $n^{2 c+2} \ell$. Let $w_{1}, \ldots, w_{N}$ be vectors in the dual lattice chosen randomly and independently from the distribution $\hat{f}$. Then with probability at least $3 / 4$,

$$
f_{W}(x) \stackrel{\text { def }}{=} \frac{1}{N} \sum_{i=1}^{N} \cos \left(2 \pi\left\langle x, w_{i}\right\rangle\right)
$$

satisfies that $\left|f_{W}(x)-f(x)\right| \leq n^{-c}$ for all $x \in L_{\ell}$.

We note that the requirement that the Fourier series is a probability measure can be somewhat relaxed. Indeed, it is easy to generalize our proof to the case in which the sum of the absolute values of the Fourier coefficients of $f$ (that is, the $l_{1}$ norm of the Fourier series) is polynomially bounded.

A closely related lemma was previously used in the work of Bruck and Smolensky [8]. There, the authors were interested in functions on the Boolean cube $\{0,1\}^{n}$. Our lemma can be seen as an adaptation of their lemma to the continuous world. Another related idea is that of truncating the small Fourier coefficients to achieve good approximation of $f$. This is done, for example, by Kushilevitz and Mansour in [21], as well as in various other contexts (e.g., signal processing). However, in those cases, one is interested in a good approximation in the $l_{2}$ norm, while here we require a good approximation in the $l_{\infty}$ norm, i.e., pointwise. ${ }^{1}$

\footnotetext{
${ }^{1}$ To demonstrate the difference between these two notions of approximation, consider a very sparse lattice. By the properties of $f$ described in Step 1, it can be seen that $f$ is essentially 0 on all except an exponentially small part of the space. In such a case, it can be shown that all the Fourier coefficients of $f$ are exponentially small. Truncating them would lead to the constant function 0 , which is a good approximation in the $l_{2}$ norm but not in the $l_{\infty}$ norm.
} 
Given this lemma, it is natural to define the witness as the list $w_{1}, \ldots, w_{N}$ of vectors in the dual lattice; this list is also referred to as $W$. We note that these vectors are typically short and hence computing them directly seems difficult.

Step 3 Here we construct an efficient NP verifier that, given $W$, verifies that a point is far from the lattice. Given a lattice $L$ and a vector $v$, it accepts if the distance of $v$ from $L$ is greater than $\sqrt{n}$ and rejects if this distance is less than $1 / 100$. This shows that $\mathrm{GapCVP}_{100 \sqrt{n}}$ is in coNP (after appropriate rescaling).

The verifier starts by performing the following test: compute $f_{W}(v)$, as defined in (1), and reject if it is at least, say, $1 / 2$. We can do this because when the distance of $v$ from $L$ is greater than $\sqrt{n}, f(v)$ is exponentially small and hence $f_{W}(v)$ must be at most $1 / \operatorname{poly}(n)<1 / 2$ (assuming the witness $W$ is chosen from $\hat{f}$ as it should be).

This verifier, however, is clearly not strong enough: the prover can 'cheat' by sending $w_{i}$ 's that have nothing to do with $\hat{f}$ or with the lattice, and for which $f_{W}(v)$ is small even though $v$ is within distance $1 / 100$ of the lattice. One might try to avoid such cheating by verifying that $f_{W}$ is close to $f$ everywhere, or, alternatively, that the $w_{i}$ 's were indeed chosen from the correct distribution $\hat{f}$. We do not know how to construct such a verifier. Instead, we provide a weaker verifier (and indeed, lose a factor of $\sqrt{\log n}$ in the approximation ratio, in comparison to what one could expect given the properties of $f$ ).

To test the witness $W$, we verify that the $w_{i}$ 's 'look like' vectors chosen from $\hat{f}$, according to some simple statistical tests. We will later see that these tests suffice to provide soundness. But what do vectors chosen from $\hat{f}$ look like? We identify two important properties. First, by definition we see that all the $w_{i}$ 's are in $L^{*}$. Second, it turns out that with high probability, for any unit vector $u \in \mathbb{R}^{n}$ it holds that $\frac{1}{N} \sum_{i=1}^{N}\left\langle u, w_{i}\right\rangle^{2}$ is bounded from above by some constant, say 3. Intuitively, this follows from the fact that the length of the $w_{i}$ 's is roughly $\sqrt{n}$ and that they are not concentrated in any particular direction. The proof uses another lemma due to Banaszczyk [7].

Fortunately, the verifier can check these two properties efficiently. The first property is easy to check by, say, solving linear equations. But how can we check the second property efficiently? It seems that we have to check it for all vectors $u$. However, we observe that we can equivalently check that the largest eigenvalue of the $n \times n$ matrix $W \cdot W^{T}$, where $W$ is the $n \times N$ matrix whose columns are the vectors $w_{1}, \ldots, w_{N}$, is at most $3 N$. Computing the eigenvalues of this matrix can be done in polynomial time.

To summarize, the verification consists of three tests. The verifier first checks that $f_{W}(v)<1 / 2$, it then checks that $W$ consists of vectors from the dual lattice, and finally, it checks that the largest eigenvalue of $W \cdot W^{T}$ is at most $3 N$. If any of these tests fails, the verifier rejects.

We now claim that the protocol is sound, by proving that any witness $W$ that passes the last two tests, satisfies $f_{W}(x) \geq 1 / 2$ for all $x$ within distance $1 / 100$ from the lattice. To see this, we note that by the definition of $f_{W}$, the fact that $W$ consists of dual vectors guarantees that the function $f_{W}$ is periodic on $L$. Indeed, for any $v \in L,\left\langle v+x, w_{i}\right\rangle=\left\langle v, w_{i}\right\rangle+\left\langle x, w_{i}\right\rangle$ with the first term being integer. Hence, it is enough to show that $f_{W}(x) \geq 1 / 2$ for any $x$ satisfying $\|x\| \leq 1 / 100$. For such $x$, the eigenvalue test implies that for most $i$ 's, $\left|\left\langle x, w_{i}\right\rangle\right|$ is small. Therefore, for such $x$ most of the cosines in the definition of $f_{W}(x)$ are close to 1. This implies that $f_{W}(x)$ is greater than $1 / 2$ and soundness follows.

Remark: It might seem that we were somewhat wasteful in Step 1. Indeed, we do not really need the function $f$ to be exponentially small; any negligible function of $n$, or even some small constant, would be good enough. So one might hope to improve the factor $\sqrt{n}$ by proving that for any point $x$ of distance at least, say, $n^{0.499}$ from the lattice, $f(x)$ is smaller than, say, $n^{-\log n}$. Unfortunately, this is false. It is known that there are lattices for which $f(x)$ is very close to 1 for points $x$ whose distance to the lattice is as large as $c \sqrt{n}$ for some constant $c>0$. See [7] for more details. 


\subsection{Another Application: The Closest Vector Problem with Preprocessing}

Steps 1 and 2 imply that important information regarding the lattice can be encoded in a short description, though this description may be very hard to find. Note that this description is independent of the target vector $v$. Hence, if we had infinite time to preprocess the lattice before seeing the vector $v$, we could prepare the approximating function $f_{W}$ and then, when given $v$, calculate $f_{W}(v)$ in polynomial time. This is exactly the setting in the Closest Vector Problem with Preprocessing (CVPP). The problem is defined as follows: given a lattice, we are allowed to preprocess it and to output a polynomially long description, without any computational restrictions on the preprocessing phase. Then, given a preprocessed lattice and a query point $v \in \mathbb{R}^{n}$, the algorithm is supposed to efficiently approximate the distance of $v$ from the lattice. The motivation for this problem comes from cryptography and coding theory. See [11] for a more precise definition and a further discussion and references. The best known inapproximability result is that CVPP is NP-hard to approximate to within a factor of $\sqrt{3}[26]$, and the best polynomial time approximation algorithm is for a factor $n$ [26]. Steps 1 and 2 in our proof immediately imply an efficient $\sqrt{n / \log n}$ approximation algorithm for CVPP.

Theorem 1.4 For any constant $c>0$, the problem GapCVPP $\sqrt{n / \log n}$ can be solved in polynomial time.

Note that by using standard methods, a solution to a gap problem can be converted to a solution to the corresponding approximation problem. Hence, the above theorem implies that for any constant $c>0$ there exists a $c \sqrt{n / \log n}$ approximation algorithm for CVPP.

\subsection{Speculation}

Note that Step 3 is not the best that one can hope for: the function $f$ has the property that it is nonnegligible in the $\sqrt{\log n}$ vicinity of lattice points. Yet, we are only able to verify that the given function $f_{W}$ is non-negligible in a constant distance. It is possible that the verification procedure can be improved so that it includes the $\sqrt{\log n}$ vicinity of lattice points. This would imply the following speculation.

\section{Speculation 1.5 GapCVP $\sqrt{n / \log n}$ is in NP $\cap$ coNP.}

Recall that this problem is currently known to be in NP $\cap$ coAM [14]. The factor $\sqrt{n / \log n}$ arises naturally in both our work (from properties of Gaussians) and in [14] (from properties of intersections of high dimensional spheres). We note that going below $\sqrt{n / \log n}$ would probably require some substantially new ideas, and in fact, might be impossible; it may be the case that this is where the NP-hardness is manifested.

\subsection{Relation to Quantum Computation}

It is intriguing to note that our result emerged from a "dequantization" of a quantum result [2], in which we showed that coGapSVP $\sqrt{n}$ is contained in the quantum analogue of the class NP, called QMA, in which both witness and verifier are quantum. In the dequantization process we replaced both witness and verifier by classical objects. This result thus continues an existing thread of quantum-inspired purely-classical results (e.g., $[19,1])$. We would like to emphasize, however, that the proof we present in the present paper is completely classical, and bares little resemblance to the original quantum proof. In fact, the new proof is stronger and holds not only for SVP but also for CVP.

\subsection{Organization}

The rest of the paper is organized as follows. Section 2 gives the basic notations and definitions. In Section 3 we define $f$ and prove its required properties. In Section 4 we prove the pointwise approximation lemma, show that $f$ satisfies the conditions of the lemma, and deduce that there exists a polynomial size circuit that 
approximates $f$. In Section 5 we show how the previous two sections imply an improved algorithm for CVPP. In Section 6 we complete the proof of the main theorem. For the sake of completeness, we add two known results in the appendices: Appendix A gives the reduction from GapSVP ${ }_{\beta}$ to $\mathrm{GapCVP}_{\beta}$, whereas Appendix $\mathrm{B}$ shows why our results (as well as previous results) imply that the lattice problems we are considering are unlikely to be NP-hard.

\section{Preliminaries}

\section{$2.1 \quad$ Lattices}

For an introduction to lattices, see [25]. A lattice in $\mathbb{R}^{n}$ is defined as the set of all integer combinations of $n$ linearly independent vectors. This set of vectors is known as a basis of the lattice and is not unique. Given a basis $\left(v_{1}, \ldots, v_{n}\right)$ of a lattice $L$, the fundamental parallelepiped is defined as

$$
\mathcal{P}\left(v_{1}, \ldots, v_{n}\right)=\left\{\sum_{i=1}^{n} x_{i} v_{i} \mid x_{i} \in[0,1)\right\} .
$$

Note that a lattice has a different fundamental parallelepiped for each possible basis. However, everything we do is independent of the basis, and so we will use the notation $\mathcal{P}(L)$ instead of $\mathcal{P}\left(v_{1}, \ldots, v_{n}\right)$. We denote by $\operatorname{det}(L)$ the volume of the fundamental parallelepiped of $L$ or equivalently, the determinant of the matrix whose columns are the basis vectors of the lattice (again, this is independent of the basis). For a point $x \in \mathbb{R}^{n}$ we define $d(x, L)$ as the minimum of $\|x-y\|$ over all $y \in L$.

For any $n$-dimensional lattice $L$, the dual lattice of $L$, denoted $L^{*}$, is an $n$-dimensional lattice defined as the set of all points in $\mathbb{R}^{n}$ with integer inner products with all lattice points,

$$
L^{*}=\left\{y \in \mathbb{R}^{n} \mid \forall x \in L\langle x, y\rangle \in \mathbb{Z}\right\} .
$$

\subsection{Shortest and Closest Vector in a Lattice}

A shortest (non-zero) vector of $L$ is a vector $x \in L$, such that $\|x\| \neq 0$ and is minimal. The following is the gap version of the shortest vector problem.

Definition 2.1 (coGapSVP) For any gap parameter $\beta=\beta(n)$ the promise problem coGapSVP ${ }_{\beta}$ is defined as follows. The input is a basis for a lattice $L$. It is a YES instance if the length of the shortest vector is more than $\beta$. It is a NO instance if the length of the shortest vector is at most 1.

We also define the gap version of the closest vector problem.

Definition 2.2 (coGapCVP) For any gap parameter $\beta=\beta(n)$ the promise problem coGapCVP ${ }_{\beta}$ is defined as follows. The input is a basis for a lattice $L$ and a vector $v$. It is a YES instance if $d(v, L)>\beta$. It is a NO instance if $d(v, L) \leq 1$.

Notice that we can replace the values $\beta$ and 1 by, say, $\beta / 100$ and $1 / 100$ respectively without really affecting the complexity of the problems. This follows from an easy reduction that simply rescales the input by a factor of 100 .

\subsection{Precision Issues}

Each vector in the input basis $v_{1}, \ldots, v_{n}$ is given with polynomially many bits. We assume that the target vector $v$ is given to us in the form $\sum a_{i} v_{i}$ where each $0 \leq a_{i}<1$ is represented by at most $\ell$ bits where $\ell=\operatorname{poly}(n)$ is some fixed global parameter. To this end we define, for a given lattice $L$, a refined lattice

$L_{\ell}=L / 2^{\ell}$. In other words, $L_{\ell}$ is given by all integer combinations of the basis vectors $\frac{1}{2^{\ell}} v_{1}, \ldots, \frac{1}{2^{\ell}} v_{n}$. Notice that we have $v \in L_{\ell}$. 


\subsection{Fourier Series and Fourier Transform}

We now describe the Fourier series and the Fourier transform including some of their basic properties. For a more in-depth treatment including proofs of some of the claims below, see, e.g., [29].

A function $f: \mathbb{R}^{n} \rightarrow \mathbb{R}$ is said to be periodic over a lattice $L$ if $f(x)=f(x+y)$ holds for all $x \in \mathbb{R}^{n}$ and for all $y \in L$. For such an $f$, one can define its Fourier series as follows. The Fourier coefficient of $f$ at $w \in L^{*}$, denoted by $\hat{f}(w)$, is defined to be

$$
\hat{f}(w)=\frac{1}{\operatorname{det}(L)} \int_{z \in \mathcal{P}(L)} f(z) e^{-2 \pi i\langle w, z\rangle} d z .
$$

(It can be shown that the above definition is independent of the basis we choose for $L$, because $f(z) e^{-2 \pi i\langle w, z\rangle}$ is periodic over $L$.) The Fourier series of $f$ at $x$ is defined by

$$
\sum_{w \in L^{*}} \hat{f}(w) e^{2 \pi i\langle w, x\rangle}
$$

Fact 2.3 For any sufficiently smooth function $f: \mathbb{R}^{n} \rightarrow \mathbb{R}$ that is periodic over some lattice $L$ and any $x \in \mathbb{R}^{n}$, the Fourier series of $f$ at $x$ is equal to $f(x)$.

The Fourier transform of a function $h: \mathbb{R}^{n} \rightarrow \mathbb{R}$ is defined as

$$
\forall w \in \mathbb{R}^{n} \quad \hat{h}(w)=\int_{\mathbb{R}^{n}} h(x) e^{-2 \pi i\langle x, w\rangle} d x .
$$

If $h: \mathbb{R}^{n} \rightarrow \mathbb{R}$ is a Gaussian, $h(x)=e^{-\pi\|x\|^{2}}$, then its Fourier transform turns out to also be a Gaussian, $\hat{h}(w)=e^{-\pi\|w\|^{2}}$.

\subsection{Some Useful Lemmas}

The following technical claim shows that all the sums that we use are well defined.

Claim 2.4 For any $n$-dimensional lattice $L$ and for any $x \in \mathbb{R}^{n}$, the sum $\sum_{y \in L} e^{-\pi\|x-y\|^{2}}$ is finite.

Proof: Notice that

$$
1=\int_{y \in \mathbb{R}^{n}} e^{-\pi\|x-y\|^{2}}=\lim _{m \rightarrow \infty}\left(\sum_{y \in L / m} e^{-\pi\|x-y\|^{2}}\right) \operatorname{det}(L / m)
$$

where $L / m$ denotes the lattice scaled down by a factor $m$. Hence, there exists an integer $m_{0}$ such that

$$
2 \geq\left(\sum_{y \in L / m_{0}} e^{-\pi\|x-y\|^{2}}\right) \operatorname{det}\left(L / m_{0}\right) .
$$

Hence, $\sum_{y \in L} e^{-\pi\|x-y\|^{2}} \leq \sum_{y \in L / m_{0}} e^{-\pi\|x-y\|^{2}}$ is finite.

We now quote two lemmas due to Banaszczyk [7] that we use throughout the proof.

Lemma 2.5 (Lemma 1.5 in [7]) For any $n$-dimensional lattice $L, x \in \mathbb{R}^{n}$ and $c>\frac{1}{\sqrt{2 \pi}}$, one has

$$
\frac{\sum_{y \in L,\|x-y\|>c \sqrt{n}} e^{-\pi\|x-y\|^{2}}}{\sum_{y \in L} e^{-\pi\|y\|^{2}}} \leq 2\left(c \sqrt{2 \pi e} \cdot e^{-\pi c^{2}}\right)^{n}=2^{-\Omega(n)} .
$$


This lemma was used in [7] to show several tight connections between a lattice and its dual (these are known as 'transference theorems'). Its proof is non-trivial; for another proof, see Štefankovič's thesis [31].

Lemma 2.6 (Lemma 1.3 in [7]) For any $n$-dimensional lattice $L$ and any unit vector $u \in \mathbb{R}^{n}$ we have

$$
\frac{\sum_{y \in L}\langle y, u\rangle^{2} e^{-\pi\|y\|^{2}}}{\sum_{y \in L} e^{-\pi\|y\|^{2}}} \leq \frac{1}{2 \pi}
$$

To get some intuition on this bound, let us mention that we can get arbitrarily close to $\frac{1}{2 \pi}$ by choosing $L$ to be a very dense lattice. In fact, it is not difficult to see that we obtain an equality if we replace sums with integrals.

\subsection{The Chernoff-Hoeffding Bound}

We will use the Chernoff-Hoeffding bound [17], which states the following. Let $X_{1}, \ldots, X_{N}$ be $N$ identically distributed independent random variables, such that for all $i, X_{i} \in[a, b]$. Then $S_{N}=\sum_{i} X_{i}$ satisfies that

$$
\operatorname{Pr}\left(\left|S_{N}-\mathrm{E}\left[S_{N}\right]\right| \geq N \varepsilon\right) \leq 2 e^{-N \varepsilon^{2} /(b-a)^{2}} .
$$

\subsection{Epsilon Nets}

Definition 2.7 Given a set $S$ in $\mathbb{R}^{n}$, we say that $A \subseteq S$ is an $\varepsilon$-net for $S$ if for every $s \in S$ there exists a point $a \in A$ such that $\|a-s\| \leq \varepsilon$.

Claim 2.8 Let $S$ be the unit sphere in $\mathbb{R}^{n}$. There exists an $\varepsilon$-net for $S$ of size at most $(2 \sqrt{n} / \varepsilon)^{n}$.

Proof: Let $C$ be $[-1,1]^{n}$, i.e., the $n$-dimensional cube of edge length 2 , and notice that $C$ contains $S$. Partition $C$ into $(2 \sqrt{n} / \varepsilon)^{n}$ small cubes of edge length $\varepsilon / \sqrt{n}$. For each small cube that intersects $S$, choose an arbitrary point in the intersection and include it in the $\varepsilon$-net. It is easy to see that the collection of these points constitutes an $\varepsilon$-net on the sphere, because any point in the sphere belongs to one of the small cubes, and the diameter of each small cube is exactly $\varepsilon$.

\section{Define $f$}

We define the function $g: \mathbb{R}^{n} \rightarrow \mathbb{R}$ as

$$
g(x)=\sum_{y \in L} e^{-\pi\|x-y\|^{2}} .
$$

This sum is finite by Claim 2.4. We then define

$$
f(x)=\frac{g(x)}{g(0)}
$$

The following lemmas show that the value of $f$ indicates the distance from the lattice.

Lemma 3.1 Let $c>\frac{1}{\sqrt{2 \pi}}$ be any constant. Then for any $x \in \mathbb{R}^{n}$, if $d(x, L) \geq c \sqrt{n}$ then $f(x) \leq 2^{-\Omega(n)}$.

Proof: The proof follows trivially from Lemma 2.5 .

Lemma 3.2 Let $c>0$ be any constant. Then for any $x \in \mathbb{R}^{n}$, if $d(x, L) \leq c \sqrt{\log n}$ then $f(x)>n^{-10 c^{2}}$. 
Proof: Notice that because of the periodicity of $f$ over the lattice, it is sufficient to prove that if $\|x\| \leq$ $c \sqrt{\log n}$ then $f(x)>n^{-10 c^{2}}$. This follows if we show that for any $x \in \mathbb{R}^{n}, f(x) \geq e^{-\pi\|x\|^{2}}$. To show this we write

$$
\begin{aligned}
g(x) & =\sum_{y \in L} e^{-\pi\|x-y\|^{2}}=\frac{1}{2} \sum_{y \in L}\left(e^{-\pi\|x-y\|^{2}}+e^{-\pi\|x+y\|^{2}}\right) \\
& =e^{-\pi\|x\|^{2}} \sum_{y \in L} e^{-\pi\|y\|^{2}} \frac{1}{2}\left(e^{-2 \pi\langle x, y\rangle}+e^{2 \pi\langle x, y\rangle}\right) \\
& \geq e^{-\pi\|x\|^{2}} \sum_{y \in L} e^{-\pi\|y\|^{2}}=e^{-\pi\|x\|^{2}} g(0)
\end{aligned}
$$

where the last inequality follows from the fact that for any positive real $r, r+\frac{1}{r} \geq 2$.

\section{Encode $f$}

Claim 4.1 The Fourier series of $f$ is given by

$$
\hat{f}(w)=\frac{e^{-\pi\|w\|^{2}}}{\sum_{z \in L^{*}} e^{-\pi\|z\|^{2}}} .
$$

Proof: By definition of $g$ and the Fourier series,

$$
\hat{g}(w)=\frac{1}{\operatorname{det}(L)} \int_{x \in \mathcal{P}(L)}\left(\sum_{y \in L} e^{-\pi\|x-y\|^{2}}\right) e^{-2 \pi i\langle x, w\rangle} d x
$$

for any $w \in L^{*}$. By the definition of $L^{*}$, we have $\langle x, w\rangle=\langle x-y, w\rangle \bmod 1$ for any $y \in L$ and so

$$
\begin{aligned}
\hat{g}(w) & =\frac{1}{\operatorname{det}(L)} \int_{x \in \mathcal{P}(L)}\left(\sum_{y \in L} e^{-\pi\|x-y\|^{2}} e^{-2 \pi i\langle x-y, w\rangle}\right) d x \\
& =\frac{1}{\operatorname{det}(L)} \int_{z \in \mathbb{R}^{n}} e^{-\pi\|z\|^{2}} e^{-2 \pi i\langle z, w\rangle} d z .
\end{aligned}
$$

This is exactly the Fourier transform of a Gaussian divided by $\operatorname{det}(L)$, and hence we have (see Subsection 2.4)

$$
\hat{g}(w)=\frac{1}{\operatorname{det}(L)} e^{-\pi\|w\|^{2}} .
$$

To derive $\hat{f}(w)$ we have to divide by $g(0)$. By Fact 2.3 ,

$$
g(0)=\sum_{w \in L^{*}} \hat{g}(w)=\frac{1}{\operatorname{det}(L)} \sum_{w \in L^{*}} e^{-\pi\|w\|^{2}},
$$

which gives us the desired result.

Corollary 4.2 The Fourier series of $f$ is a probability measure on the dual lattice (i.e., it is non-negative and the sum over all points in the dual lattice is 1$)$.

We are thus in a situation which satisfies the conditions of Lemma 1.3. It remains to prove the lemma.

Proof of Lemma 1.3: By the conditions of the lemma, the Fourier coefficients of $f$ are non-negative and their sum is 1 . We apply Fact 2.3 and obtain

$$
f(x)=\sum_{w \in L^{*}} \hat{f}(w) e^{2 \pi i\langle w, x\rangle}=\sum_{w \in L^{*}} \hat{f}(w) \cos (2 \pi\langle w, x\rangle)
$$


where the last equality follows from the fact that both $f$ and $\hat{f}$ are real, and so the imaginary part cancels out. Hence $f(x)$ can be seen as the expectation of $\cos (2 \pi\langle w, x\rangle)$ (whose values range between -1 and 1 ), where $w$ is chosen according to the probability measure $\hat{f}$,

$$
f(x)=\mathrm{E}_{w \sim \hat{f}}[\cos (2 \pi\langle w, x\rangle)] .
$$

Let $x \in \mathbb{R}^{n}$. By the Chernoff-Hoeffding bound, (2), we have that the probability that the mean of $N$ samples is not within a window of $n^{-c}$ of the correct expectation is $2^{-\Omega\left(N / n^{2 c}\right)}$. We now want to show that this holds simultaneously for all $x \in L_{\ell}$. Since $f$ is periodic over the lattice, it suffices to consider $x$ in $\mathcal{P}(L) \cap L_{\ell}$. By definition of $L_{\ell}$, there are exactly $2^{\ell n}$ such points. Hence, by the union bound, the probability that the approximation is within $n^{-c}$ window of the correct expectation at all points in $L_{\ell}$ simultaneously is at least $1-2^{n \ell} 2^{-\Omega\left(N / n^{2 c}\right)}$. Since $N=n^{2 c+2} \ell$ we get exponentially good confidence.

Applying the lemma in our case implies that with high probability, $f_{W}$ approximates $f$ everywhere in $L_{\ell}$ to within polynomial precision. In particular, since $v \in L_{\ell}$, we have that $f_{W}(v)$ approximates $f(v)$ to within polynomial precision.

Remark: In fact, the above lemma is stronger than what we need for our main application, namely for the proof of Theorem 1.1. We will only need the lemma to hold for any given $x$, but not necessarily simultaneously for all $x \in L_{\ell}$, and so for our main application the final union bound in the proof is unnecessary. However, for the CVPP application, which follows next, we need the full strength of the above lemma.

\section{Interlude: The Closest Vector Problem with Preprocessing}

Proof of Theorem 1.4: Let $c>0$ be an arbitrary constant. By Lemma 1.3, there exists some $N=\operatorname{poly}(n)$ and a sequence $w_{1}, \ldots, w_{N}$ such that the function $f_{W}$ defined by them approximates $f$ at any point in $L_{\ell}$ to within $\frac{1}{4} n^{-10 / c^{2}}$. Given a lattice $L$, the preprocessing step outputs such a sequence $w_{1}, \ldots, w_{N}$. Given a vector $v$ and the preprocessed lattice $w_{1}, \ldots, w_{N}$, the computation step involves a simple computation of $f_{W}(v)$. If its value is more than $\frac{1}{2} n^{-10 / c^{2}}$ then we decide that $d(x, L) \leq \sqrt{\log n} / c$; otherwise, we decide that $d(x, L)>\sqrt{n}$. The correctness of the algorithm follows from Lemmas 3.1, 3.2 and 1.3.

\section{Verify $f_{W}$}

In this section we prove Theorem 1.1 by showing that GapCVP $\mathrm{P}_{100 \sqrt{n}}$ is in coNP. We do this by providing a coNP verifier for a rescaled problem, where the NO instances have distance at most 1/100 from $L$, and the YES instances have distance more than $\sqrt{n}$ from $L$. The witness is a sequence of vectors $w_{1}, \ldots, w_{N}$, where $N$ is chosen to be a large enough polynomial in $n$, say, $N=n^{4} \ell$. It will be convenient to refer to the witness, equivalently, as an $n \times N$ matrix $W$ whose columns correspond to $w_{1}, \ldots, w_{N}$.

The verifier performs three tests and accepts if and only if all of them are satisfied:

(a) Checks that $f_{W}(v)<1 / 2$,

(b) Checks that the $w_{i}$ 's are in the dual lattice $L^{*}$,

(c) Checks that the maximal eigenvalue of the $n \times n$ positive semidefinite matrix $W W^{T}$ is at most $3 N$.

It is easy to see that the verifier can be implemented in polynomial time. 


\subsection{Soundness}

Assume that $v$ is a NO instance, i.e., its distance from $L$ is at most 1/100 and assume that tests $(b),(c)$ accept. We will show that test $(a)$ must reject. First, since test $(b)$ accepts, we have that $f_{W}$ is periodic over $L$. Let $\tau(v)$ denote the vector given by $v$ minus the lattice point closest to $v$. Notice that $\|\tau(v)\| \leq 1 / 100$. Since $f_{W}$ is periodic on the lattice, $f_{W}(v)=f_{W}(\tau(v))$. It thus suffices to prove that $f_{W}(\tau(v)) \geq 1 / 2$, or, for that matter, that $f_{W}(x) \geq 1 / 2$ for all $x$ in a ball of radius $1 / 100$ around the origin.

This is done as follows. Let $x$ be such that $\|x\| \leq 1 / 100$. Since test $(c)$ accepts, we have that

$$
\frac{1}{N} \sum_{j=1}^{N}\left\langle x, w_{j}\right\rangle^{2}=\frac{1}{N} x^{T} W W^{T} x \leq \frac{1}{N} \frac{3 N}{10000}=\frac{3}{10000}
$$

where the inequality follows by expressing $x$ in the eigenvector basis of $W W^{T}$. Using the inequality $\cos x \geq$ $1-x^{2} / 2$ (valid for any $x \in \mathbb{R}$ ) we get

$$
f_{W}(x)=\frac{1}{N} \sum_{j=1}^{N} \cos \left(2 \pi\left\langle x, w_{j}\right\rangle\right) \geq 1-\frac{4 \pi^{2}}{2 N} \sum_{j=1}^{N}\left\langle x, w_{j}\right\rangle^{2} \geq 1-\frac{6 \pi^{2}}{10000}>\frac{1}{2} .
$$

\subsection{Completeness}

Suppose $v$ is a YES instance, i.e., its distance from $L$ is at least $\sqrt{n}$. We show that a random witness chosen according to $\hat{f}$ satisfies each of the above tests with probability at least $3 / 4$. Clearly, this implies the existence of a witness that satisfies all tests. Test $(b)$ is always satisfied because $\hat{f}$ 's support is on $L^{*}$.

Claim 6.1 The probability that a random witness chosen according to $\hat{f}$ satisfies test $(a)$ is more than $3 / 4$, i.e., $f_{W}(v)<1 / 2$ with probability at least $3 / 4$.

Proof: The proof follows trivially from Lemma 3.1 combined with Lemma 1.3.

For the proof that test $(c)$ is satisfied, we need the following geometrical lemma.

Lemma 6.2 Let $\delta, K, r$ be some positive numbers and let $D$ be a distribution on $\mathbb{R}^{n}$ such that for any fixed unit vector $u$,

$$
\mathrm{E}_{w \sim D}\left[\langle u, w\rangle^{2}\right] \leq r^{2}
$$

and, moreover,

$$
\operatorname{Pr}_{w \sim D}(\|w\| \geq K r)<\delta .
$$

Let $W=\left[w_{1}, \ldots, w_{N}\right]$ be a matrix obtained by picking each column independently at random according to distribution $w_{i} \sim D$. Then, with probability at least $1-2 e^{-N / K^{4}}\left(4 \sqrt{n} K^{2}\right)^{n}-N \delta$ (over the choice of matrix $W)$ the maximum eigenvalue of the $n \times n$ matrix $W W^{T}$ is at most $3 \mathrm{Nr}^{2}$.

Proof: The largest eigenvalue of $W \cdot W^{T}$ is at most $3 N r^{2}$ if and only if

$$
\frac{1}{N} \sum_{i=1}^{N}\left\langle u, w_{i}\right\rangle^{2} \leq 3 r^{2}
$$

for all unit vectors $u \in \mathbb{R}^{n}$. In the following, we show that this condition is satisfied with the desired probability. Let $\xi: \mathbb{R}^{n} \rightarrow \mathbb{R}^{n}$ be the function defined by $\xi(x)=x$ if $\|x\| \leq K r$ and $\xi(x)=0$ otherwise. Clearly, for any unit vector $u$,

$$
\mathrm{E}_{w \sim D}\left[\langle u, \xi(w)\rangle^{2}\right] \leq \mathrm{E}_{w \sim D}\left[\langle u, w\rangle^{2}\right] \leq r^{2} .
$$


Moreover, the random variable $\langle u, \xi(w)\rangle^{2}$ takes values in the interval $\left[0,(K r)^{2}\right]$. Hence, the ChernoffHoeffding bound (2) implies that for fixed any unit vector $u$, a sequence of samples $w_{1}, \ldots, w_{N}$ from $D$ satisfies

$$
\frac{1}{N} \sum_{i=1}^{N}\left\langle u, \xi\left(w_{i}\right)\right\rangle^{2} \leq 2 r^{2}
$$

with probability at least $1-2 e^{-N / K^{4}}$.

We now need to extend the argument to hold for all $u$ 's simultaneously. Let $\varepsilon=\frac{1}{2} K^{-2}$. By Claim 2.8, there exists an $\varepsilon$-net $A$ on the unit sphere containing at most $(2 \sqrt{n} / \varepsilon)^{n}$ points. We now apply the union bound on the set of all vectors $u$ in $A$. It follows that (3) holds with probability at least $1-2 e^{-N / K^{4}}\left(4 \sqrt{n} K^{2}\right)^{n}$ for all $u \in A$ simultaneously.

Next, we show that if (3) holds for all $u \in A$, then a slightly weaker version of it holds for all unit vectors. Consider an arbitrary unit vector $u^{\prime}$. Let $u \in A$ be the closest point to $u^{\prime}$ in $A$. Notice that $\left\|u-u^{\prime}\right\| \leq \varepsilon$. Thus,

$$
\begin{aligned}
\left|\frac{1}{N} \sum_{i=1}^{N}\left\langle u^{\prime}, \xi\left(w_{i}\right)\right\rangle^{2}-\frac{1}{N} \sum_{i=1}^{N}\left\langle u, \xi\left(w_{i}\right)\right\rangle^{2}\right| & \leq \frac{1}{N} \sum_{i=1}^{N}\left|\left\langle u^{\prime}-u, \xi\left(w_{i}\right)\right\rangle\left\langle u^{\prime}+u, \xi\left(w_{i}\right)\right\rangle\right| \\
& \leq 2 \varepsilon \max _{i}\left\|\xi\left(w_{i}\right)\right\|^{2} \leq 2 \varepsilon(K r)^{2}=r^{2} .
\end{aligned}
$$

This yields that with probability at least $1-2 e^{-N / K^{4}}\left(4 \sqrt{n} K^{2}\right)^{n}$ over the choice of the $w_{i}$ 's it holds that

$$
\frac{1}{N} \sum_{i=1}^{N}\left\langle u, \xi\left(w_{i}\right)\right\rangle^{2} \leq 2 r^{2}+r^{2}=3 r^{2}
$$

for all unit vectors $u$. It remains to notice that with probability at least $1-N \delta, \xi\left(w_{i}\right)=w_{i}$ for all $i$.

Lemma 6.3 The probability that a random witness chosen according to $\hat{f}$ satisfies test $(c)$ is at least $3 / 4$.

Proof: According to Lemma 2.5, the probability that the norm of a vector chosen from $\hat{f}$ is more than, say, $\sqrt{n}$, is $2^{-\Omega(n)}$. Moreover, Lemma 2.6 states that for any unit vector $u$, the average norm squared of the projection on $u$ of a vector $w$ chosen from $\hat{f}$ is at most $\frac{1}{2 \pi}$,

$$
\mathrm{E}_{w \sim \hat{f}}\left[\langle u, w\rangle^{2}\right] \leq \frac{1}{2 \pi} .
$$

We now apply Lemma 6.2 with $r=1, K=\sqrt{n}$, and $\delta=2^{-\Omega(n)}$. This yields that the maximum eigenvalue of $W \cdot W^{T}$ is at most $3 N$ with probability at least $1-2^{-\Omega\left(n^{2}\right)}$.

\section{Acknowledgements}

We thank Daniele Micciancio and the anonymous referees for helpful comments.

\section{References}

[1] S. Aaronson. Lower bounds for local search by quantum arguments. In Proc. 36th ACM Symp. on Theory of Computing (STOC), pages 465-474. ACM, 2004.

[2] D. Aharonov and O. Regev. A lattice problem in quantum NP. In Proc. 44th Annual IEEE Symp. on Foundations of Computer Science (FOCS), pages 210-219. IEEE, 2003.

[3] M. Ajtai. Generating hard instances of lattice problems (extended abstract). In Proc. 28th ACM Symp. on Theory of Computing (STOC), pages 99-108. ACM, 1996. 
[4] M. Ajtai. The shortest vector problem in $l_{2}$ is NP-hard for randomized reductions (extended abstract) 10-19. In Proc. 30th ACM Symp. on Theory of Computing (STOC), pages 10-19. ACM, 1998.

[5] M. Ajtai and C. Dwork. A public-key cryptosystem with worst-case/average-case equivalence. In Proc. 29th ACM Symp. on Theory of Computing (STOC), pages 284-293. ACM, 1997.

[6] M. Ajtai, R. Kumar, and D. Sivakumar. A sieve algorithm for the shortest lattice vector problem. In Proc. 33rd ACM Symp. on Theory of Computing, pages 601-610. ACM, 2001.

[7] W. Banaszczyk. New bounds in some transference theorems in the geometry of numbers. Mathematische Annalen, 296(4):625-635, 1993.

[8] J. Bruck and R. Smolensky. Polynomial threshold functions, $\mathrm{AC}^{0}$ functions, and spectral norms. SIAM J. Comput., 21(1):33-42, 1992.

[9] J.-Y. Cai and A. Nerurkar. A note on the non-NP-hardness of approximate lattice problems under general Cook reductions. Inform. Process. Lett., 76(1-2):61-66, 2000.

[10] I. Dinur, G. Kindler, R. Raz, and S. Safra. Approximating CVP to within almost-polynomial factors is NP-hard. Combinatorica, 23(2):205-243, 2003.

[11] U. Feige and D. Micciancio. The inapproximability of lattice and coding problems with preprocessing. In Computational Complexity, pages 44-52. IEEE, 2002.

[12] C. F. Gauss. Disquisitiones Arithmeticae. Gerh. Fleischer Iun, 1801.

[13] O. Goldreich. A comment available online at http://www.wisdom. weizmann.ac.il/ oded/p_lp.html.

[14] O. Goldreich and S. Goldwasser. On the limits of nonapproximability of lattice problems. J. Comput. System Sci., 60(3):540-563, 2000.

[15] O. Goldreich, D. Micciancio, S. Safra, and J.-P. Seifert. Approximating shortest lattice vectors is not harder than approximating closest lattice vectors. Inform. Process. Lett., 71(2):55-61, 1999.

[16] J. Håstad, B. Just, J. C. Lagarias, and C.-P. Schnorr. Polynomial time algorithms for finding integer relations among real numbers. SIAM J. Comput., 18(5):859-881, 1989.

[17] W. Hoeffding. Probability inequalities for sums of bounded random variables. Journal of the American Statistical Association, 58:13-30, 1963.

[18] R. Kannan. Improved algorithms for integer programming and related lattice problems. In Proc. $15^{\text {th }}$ Symp. Theory. of Comp., pages 193-206, 1983.

[19] I. Kerenidis and R. de Wolf. Exponential lower bound for 2-query locally decodable codes via a quantum argument. In Proc. 35th ACM Symp. on Theory of Computing (STOC), pages 106-115, 2003.

[20] S. Khot. Hardness of approximating the shortest vector problem in lattices. In Proc. 45th Annual IEEE Symp. on Foundations of Computer Science (FOCS), pages 126-135, 2004.

[21] E. Kushilevitz and Y. Mansour. Learning decision trees using the fourier spectrum. SIAM J. Comput., 22(6):1331-1348, 1993.

[22] J. C. Lagarias, H. W. Lenstra, Jr., and C.-P. Schnorr. Korkin-Zolotarev bases and successive minima of a lattice and its reciprocal lattice. Combinatorica, 10(4):333-348, 1990.

[23] A. K. Lenstra, H. W. Lenstra, and L. Lovász. Factoring polynomials with rational coefficients. Math. Ann., 261:515-534, 1982. 
[24] D. Micciancio. The shortest vector problem is NP-hard to approximate to within some constant. SIAM Journal on Computing, 30(6):2008-2035, Mar. 2001. Preliminary version in FOCS 1998.

[25] D. Micciancio and S. Goldwasser. Complexity of Lattice Problems: a cryptographic perspective, volume 671 of The Kluwer International Series in Engineering and Computer Science. Kluwer Academic Publishers, Boston, Massachusetts, Mar. 2002.

[26] O. Regev. Improved inapproximability of lattice and coding problems with preprocessing. In Proc. of 18th IEEE Annual Conference on Computational Complexity (CCC), pages 363-370, 2003.

[27] C.-P. Schnorr. A hierarchy of polynomial time lattice basis reduction algorithms. Theoretical Computer Science, 53(2-3):201-224, 1987.

[28] C.-P. Schnorr. Factoring integers and computing discrete logarithms via diophantine approximation. In Proc. of Eurocrypt '91, volume 547, pages 171-181. Springer-Verlag, 1991.

[29] E. M. Stein and G. Weiss. Introduction to Fourier analysis on Euclidean spaces. Princeton University Press, Princeton, N.J., 1971. Princeton Mathematical Series, No. 32.

[30] P. van Emde Boas. Another NP-complete problem and the complexity of computing short vectors in a lattice. Technical report, University of Amsterdam, Department of Mathematics, Netherlands, 1981. Technical Report 8104.

[31] D. Štefankovič. Fourier transforms in computer science. Master's Thesis, University of Chicago, Department of Computer Science, TR-2002-03.

\section{A Reducing GapSVP to GapCVP}

Lemma A.1 If for some $\beta=\beta(n)$, GapCVP $_{\beta}$ is in coNP then so is $\mathrm{GapSVP}_{\beta}$.

Proof: Consider an instance of $\operatorname{GapSVP}_{\beta}$ given by the lattice $L$ whose basis is $\left(b_{1}, \ldots, b_{n}\right)$. We map it to $n$ instances of $\mathrm{GapCVP}_{\beta}$ where the $i$ th instance, $i=1, \ldots, n$, is given by the lattice $L_{i}$ spanned by $\left(b_{1}, \ldots, b_{i-1}, 2 b_{i}, b_{i+1}, \ldots, b_{n}\right)$ and the target vector $b_{i}$. In the following we show that this mapping has the property that if $L$ is a YES instance of $\operatorname{GapSVP}_{\beta}$ then at least one of $\left(L_{i}, b_{i}\right)$ is a YES instance of GapCVP $\beta$ and if $L$ is a NO instance then all $n$ instances $\left(L_{i}, b_{i}\right)$ are NO instances. This will complete the proof of the lemma since a NO witness for $L$ can be given by $n$ NO witnesses for $\left(L_{i}, b_{i}\right)$.

Consider the case where $L$ is a YES instance. In other words, if

$$
u=a_{1} b_{1}+a_{2} b_{2}+\cdots+a_{n} b_{n}
$$

denotes the shortest vector, then its length is at most 1 . Notice that not all the $a_{i}$ 's are even for otherwise the vector $u / 2$ is a shorter lattice vector. Let $j$ be such that $a_{j}$ is odd. Then the distance of $b_{j}$ from the lattice $L_{j}$ is at most $\|u\| \leq 1$ since $b_{j}+u \in L_{j}$. Hence, $\left(L_{j}, b_{j}\right)$ is a YES instance of GapCVP ${ }_{\beta}$. Now consider the case where $L$ is a NO instance of $\operatorname{GapSVP}_{\beta}$. That is, the length of the shortest vector is more than $\beta$. Since for any $i \in[n], b_{i} \notin L_{i}$ this implies that $d\left(b_{i}, L_{i}\right)>\beta$. Hence, $\left(L_{i}, b_{i}\right)$ is a NO instance of GapCVP ${ }_{\beta}$.

\section{B GapSVP $\sqrt{n}$ and GapCVP $\sqrt{n}$ are unlikely to be NP-hard}

It is easy to see that Theorem 1.1 implies that if GapSVP ${ }_{c \sqrt{n}}$ or GapCVP ${ }_{c \sqrt{n}}$ are NP-hard under Karp reductions then NP $\subseteq$ coNP and the polynomial hierarchy collapses ( $c$ is the constant from that theorem). In this section we show that the same is true for Cook reductions. 
This does not follow immediately from our main theorem. Indeed, there is nothing special about a problem in coNP being NP-hard under Cook reductions (for example, coSAT is such a problem). However, in our case, the problem in question, namely GapCVP ${ }_{c \sqrt{n}}$, is also known to be in NP. We might now hope to show that if a problem in NP $\cap$ coNP is NP-hard under Cook reductions, then the polynomial hierarchy collapses. This implication is not too difficult to show for total problems (i.e., languages). However, we are dealing with promise problems and for such problems this implication is not known to hold. In a nutshell, the difficulty arises because a Cook reduction might perform queries that are neither a YES instance nor a NO instance and for such queries we have no witness.

This issue can be resolved by using the fact that not only GapCVP ${ }_{c \sqrt{n}} \in$ NP but also CVP $\in$ NP (and similarly for SVP). In other words, no promise is needed in order to show that a point is close to the lattice. In the following, we will show a proof that holds for any problem with the above properties. We remark that a similar proof has already appeared before (see $[25,9,13])$ and we repeat it here mainly for completeness.

Lemma B.1 Let $\Pi=\left(\Pi_{\mathrm{YES}}, \Pi_{\mathrm{NO}}\right)$ be a promise problem and let $\Pi_{\mathrm{MAYBE}}$ denote all instances outside $\Pi_{\mathrm{YES}} \cup$ $\Pi_{\mathrm{NO}}$. Assume that $\Pi$ is in coNP and that the (non-promise) problem $\Pi^{\prime}=\left(\Pi_{\mathrm{YES}} \cup \Pi_{\mathrm{MAYBE}}, \Pi_{\mathrm{NO}}\right)$ is in $\mathrm{NP}$. Then, if $\Pi$ is NP-hard under Cook reductions then NP $\subseteq$ coNP and the polynomial hierarchy collapses.

Proof: Assume there exists a Cook reduction from, say, SAT to П. That is, there exists a polynomial time procedure $T$ that solves SAT given access to an oracle for $\Pi$. Notice that while the oracle is guaranteed to answer YES on queries from $\Pi_{Y E S}$ and NO on queries from $\Pi_{N O}$, its answers on queries from $\Pi_{\mathrm{MAYBE}}$ are arbitrary and should not affect the output of $T$.

Since $\Pi \in$ coNP, there exists a verifier $V_{1}$ and a witness $w_{1}(x)$ for every $x \in \Pi_{\mathrm{NO}}$ such that $V_{1}$ accepts $\left(x, w_{1}(x)\right)$. Moreover, $V_{1}$ rejects $(x, w)$ for any $x \in \Pi_{Y E S}$ and any $w$. Similarly, since $\Pi^{\prime} \in \mathrm{NP}$, there exists a verifier $V_{2}$ and a witness $w_{2}(x)$ for every $x \in \Pi_{\text {YES }} \cup \Pi_{\text {MAYBE }}$ such that $V_{2}$ accepts $\left(x, w_{2}(x)\right)$. Moreover, $V_{2}$ rejects $(x, w)$ for any $x \in \Pi_{\text {NO }}$ and any $w$.

We would like to show that SAT is in coNP. Let $\Phi$ be a SAT instance and let $x_{1}, \ldots, x_{k}$ be the set of oracle queries which $T$ performs on input $\Phi$. Our witness consists of $k$ pairs, one for each $x_{i}$. For $x_{i} \in \Pi_{\text {NO }}$ we include the pair (NO, $\left.w_{1}\left(x_{i}\right)\right)$ and for $x_{i} \in \Pi_{\text {YES }} \cup \Pi_{\text {MAYBE }}$ we include the pair $\left(\mathrm{YES}, w_{2}\left(x_{i}\right)\right.$ ). The verifier simulates $T$; for each query $x_{i}$ that $T$ performs, the verifier reads the pair corresponding to $x_{i}$ in the witness. If the pair is of the form (YES, $w$ ) then the verifier checks that $V_{2}\left(x_{i}, w\right)$ accepts and then returns YES to $T$. Similarly, if the pair is of the form $(\mathrm{NO}, w)$ then the verifier checks that $V_{1}\left(x_{i}, w\right)$ accepts and then returns NO to $T$. If any of the calls to $V_{1}$ or $V_{2}$ rejects, then the verifier rejects. Finally, if $T$ outputs that $\Phi$ is satisfiable, the verifier rejects and otherwise it accepts.

The completeness follows easily. More specifically, if $\Phi$ is unsatisfiable then the witness described above will cause the verifier to accept. In order to prove soundness, assume that $\Phi$ is satisfiable and let us show that the verifier rejects. Notice that for each query $x_{i} \in \Pi_{\mathrm{NO}}$ the witness must include a pair of the form $(N O, w)$ because otherwise $V_{2}$ would reject. Similarly, for each query $x_{i} \in \Pi_{Y E S}$ the witness must include a pair of the form (YES, $w$ ) because otherwise $V_{1}$ would reject. This implies that $T$ receives the correct answers for all of its queries inside $\Pi_{N O} \cup \Pi_{Y E S}$ and must therefore output the correct answer, i.e., that $\Phi$ is satisfiable and then the verifier rejects. 